\title{
Congenital Limb Anomaly as a New Presentation of Arthrogryposis-Renal Problems-Cholestasis (ARC) Syndrome in an Iranian Infant: A Case Report
}

\author{
Mitra Basiratnia ${ }^{1}$, Forough Saki ${ }^{\circledR}$ \\ ${ }^{1}$ Shiraz Nephrology-Urology Research Center, Shiraz University of Medical Sciences, Shiraz, Iran. \\ ${ }^{2}$ Endocrinology and Metabolism Research Center, Shiraz University of Medical Sciences, Shiraz, Iran.
}

\begin{abstract}
Background: Arthrogryposis-Renal Problems-Cholestasis (ARC) syndrome is a rare autosomal recessive disease mostly presenting with arthrogryposis, renal problems and cholestasis. We present the first report of ARC syndrome in Iran manifested.Case Report: A 4-month-old male infant was admitted in our hospital with jaundice, poor feeding, tachypnea and lethargy. He has a history of congenital limb anomaly in his feet and jaundice on fifth day of birth. Laboratory and imaging studies revealed renal tubular acidosis, nephrocalcinosis and cholestasis. Despite antibiotic therapy, he died at the third day of hospitalization due to sepsis of pseudomonas aeroginosa and Escherichia coli co-infection and upper gastrointestinal bleeding secondary to coagulopathy of liver failure.Conclusion: ARC syndrome has various and rare clinical manifestations such as lower limb congenital anomaly, hypothyroidism, liver failure and nephrocalcinosis.[GMJ. 2015;4(3):117-21]
\end{abstract}

Keywords: Lower limb anomaly; Arthrogryposis-Renal Problems-Cholestasis (ARC) syndrome; Hypothyroidism

\section{Introduction}

A rthrogryposis, Renal problems and Cholestasis (ARC) syndrome is a rare autosomal recessive disorder; it was first described in 1973 in a consanguineous family by Lutz-Richner and Landolt [1]. Due to variability in signs and symptoms, they have been classified into complete and incomplete forms [2]. Up to now, about 60 cases have been reported in the literature. Other clinical manifestations of ARC syndrome include ichthyosis, congenital heart disease, cerebral malformation, sensory hearing loss, diarrhea, recurrent

\section{GMJ}

2013 Galen Medical Journal

Fax: +98 7312227091

PO Box 7461686688

Email:info@gmj.ir febrile illness and abnormal platelet morphology and function $[3,4]$.We present the first report of ARC syndrome in Iran manifested with arthrogryposis, renal problem, cholestasis and liver failure, hypothyroidism, congenital limb anomaly and ichthyosis. In addition, we compare our case with other reports of this syndrome published to date.

\section{Case Presentation}

A 4-month-old male infant was admitted in our hospital with jaundice, poor feeding, tachypnea and lethargy. He was the second

\footnotetext{
Correspondence to:

Forough Saki, Endocrinology and Metabolism Research Center, Shiraz University of Medical Sciences, Shiraz, Iran.

Telephone Number: (+98) 71-36473096

Email Address :sakeif@sums.ac.ir
} 
child of consanguineous parents and product of cesarean section due to breech presentation at term, after an uneventful pregnancy. His first and fifth minutes Apgar score were good. His birth weight was $2700 \mathrm{~g}$, birth length was $49 \mathrm{~cm}$ and birth head circumference was 34 $\mathrm{cm}$. He had a congenital limb anomaly in his feet (absence of one toe and overriding of the 1 st and 4 th toes on the 2 nd and 3rd ones) (figure 1). He also had dry and scaly skin, jaundice and flexion contracture in both upper and lower extremities (figure 2). Five days after birth, he developed jaundice and clayed color stool that slightly increased in the following days, so he was referred to a physician when he was 20 days old with yellowish skin and sclera and mild hepatomegaly; laboratory examination showed mild normal anion gap metabolic acidosis and direct hyperbilirubinemia (serum total bilirubin level was $21.5 \mathrm{mg} /$ $\mathrm{dl}$; conjugated bilirubin level was $18.1 \mathrm{mg} /$ dl). Serum level of aspartate aminotransferase (AST) was $55 \mathrm{Iu} / \mathrm{L}$, alanine aminotransferase (ALT) was Iu/L, gamma glutamyltransferase (GGT) was $7 \mathrm{Iu} / \mathrm{L}$, Alkaline phosphatase was $454 \mathrm{Iu} / \mathrm{L}$ and Albumin was $3.5 \mathrm{mg} / \mathrm{dl}$. Thyroid function test revealed $\mathrm{TSH}=10.39 \mu \mathrm{Iu} / \mathrm{ml}, \mathrm{T} 3=$ $0.3 \mathrm{nmol} / \mathrm{L}$ and T4=48 nmol/L. Microbiologic studies for antibodies against toxoplasmosis, cytomegalovirus (CMV), rubella and herpes simplex; antigen and antibodies against hepatitis viruses $\mathrm{A}, \mathrm{B}$ and $\mathrm{C}$ were negative. Urine and blood cultures were negative. Plasma alpha-1-antitrypsin level and sweat chloride tests were normal. Analysis of serum amino acids was normal. Complete blood count was normal. Hepatobiliary scan with TC $99 \mathrm{~m}$ showed good liver uptake without appropriate excretion even 24 hours post-injection that was in favor of biliary atresia. Abdominal sonography showed hepatomegaly with normal echogenicity. On 37 days of life, exploratory laparatomy, intra-operative cholangiography and liver biopsy were done for him. It revealed patent hepatic and common bile ducts and no sign of biliary atresia. Liver biopsy demonstrated deposition of brown-yellowish pigments in hepatocytes especially granular types (figure 3). In addition, it showed lobular disarray and giant cell transformation and severe cholestasis. Portal tracts were unremark- able. There was no fibrosis. Bile duct numbers were adequate. So, the patient was treated with levothyroxin, phenobarbital, multivitamin and ursodeoxycholic acid.

Two months later (when he was 4 month old), he developed poor feeding, tachypnea, lethargyness and diarrhea, so he was referred to our hospital for further evaluation. On physical examination, he had dry yellowish and scaly skin with decreased subcutaneous fat tissue and was lethargic. He also had edematous extremities and scrotum. Body temperature was $36.4^{\circ} \mathrm{c}$, heart rate was 110 beats/ minute, respiratory rate was 64 per minute, weight was $3220 \mathrm{~g}$, head circumference was $35 \mathrm{~cm}$ and length was $55 \mathrm{~cm}$. He had lowset ears. His liver was palpated $4 \mathrm{~cm}$ below the costal margin. Laboratory investigations showed electrolyte imbalance (serum $\mathrm{Na}=164$ $\mathrm{meq} / \mathrm{L}, \mathrm{K}=3.1 \mathrm{meq} / \mathrm{L}, \mathrm{Cl}=131 \mathrm{meq} / \mathrm{L}, \mathrm{Cal}-$ cium $=7.1 \mathrm{mg} / \mathrm{dl}, \mathrm{Ph}=5.4 \mathrm{mg} / \mathrm{dl}$, Albumin $=2.1 \mathrm{mg} / \mathrm{dl}, \mathrm{BUN}=54 \mathrm{mg} / \mathrm{dl}$ and $\mathrm{Cr}=1.5 \mathrm{mg} /$ dl). Also, he had normal anion gap metabolic acidosis $(\mathrm{Hco} 3=12 \mathrm{meq} / \mathrm{L}$, Anion gap $=12$. Urine analysis showed $2+$ proteinuria. The measured urine calcium to creatinine ratio was 2.7 and urine albumin to creatinine ratio was 0.8 . Urine specific gravity was 1.006 and urine output was $6 \mathrm{cc} / \mathrm{kg} / \mathrm{hr}$. Serum PT and PTT were prolonged. Abdominal sonography showed increased parenchymal echogenicity of both kidneys more in the medulla typically seen in medullary nephrocalcinosis. Parenteral fluid, electrolyte, fresh frozen plasma, bicarbonate and broad spectrum antibiotic

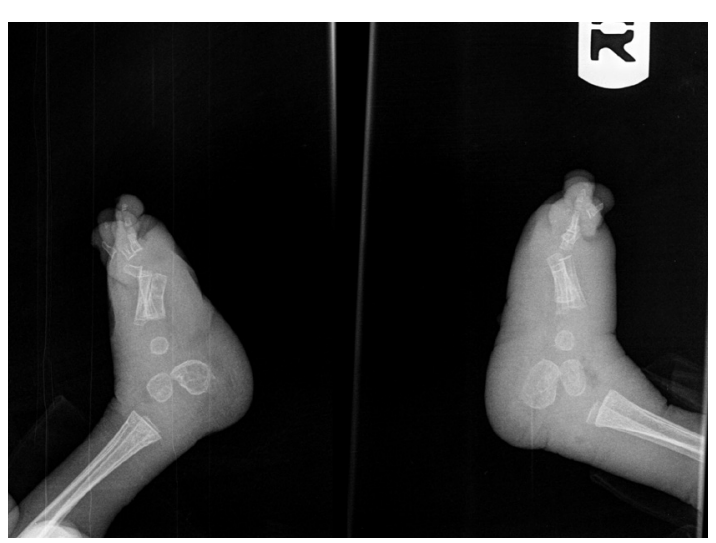

Figure 1. X-ray of the right and left feet showed absence of one toe. 


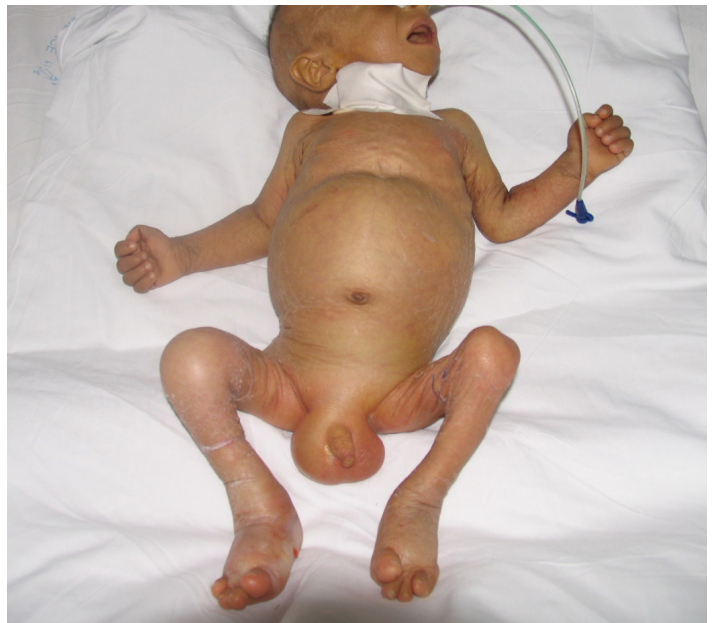

Figure 2. This is the presented four-month infant with ARC syndrome. Note the absence of one toe in the feet and overriding of the first and forth toe on the second and third one, also note the low set ears, edema of extremities and flexion contracture of the knees.

therapy were started to correct metabolic acidosis, dehydration coagulopathy and infection. Unfortunately, he died at the third day of hospitalization due to sepsis of pseudomonas aeroginosa and Escherichia coli co-infection and upper gastrointestinal bleeding secondary to coagulopathy of liver failure. His parents did not agree with genetic studies. Their first child had died due to prolonged jaundice in the second month of life due to an unknown cause.

\section{Discussion}

ARC syndrome is a hereditable fetal disease with a wide range of clinical manifestation [5]. It has three key presentations including arthrogryposis, renal tubular dysfunction and cholestasis [3]. Nearly all 60 patients with ARC syndrome that have been reported since 1973 had consanguineous parents and autosomal recessive inheritance [6,7]. Most of the reported patients with ARC syndromes were from Pakistan [3], but some have been reported from Saudi Arabia [3,4], Turkey [5,6], United Kingdom [8,9], Italy[4], Portugal [4] and Iran [2].

We report the third case of ARC syndrome in Iran [2], which manifested with arthrogry-

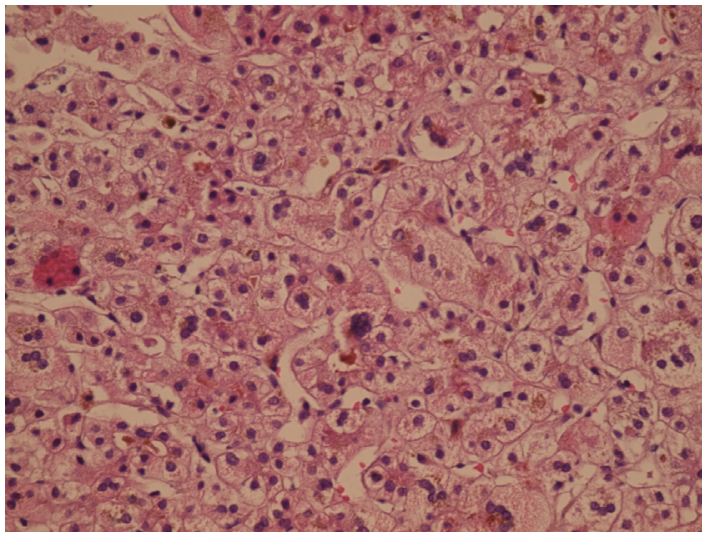

Figure 3. Liver biopsy showed lobular disarray, giant cell transformation of the hepatocytes; severe cholestasis and granular type brownyellowish pigments in the hepatocytes.

posis, renal problem, cholestasis, diarrhea, failure to thrive and dry skin. In addition, our case is the first case presented with congenital lower limb anomaly. One of the most common manifestations of ARC syndrome is arthrogryposis which was present at birth in our case. This anomaly might be due to rarefaction of the motor neurons in the anterior horn of the spinal cord [6]. It may include talipes equinovarus, radial deviation of the wrists, club feet and hip dislocation. Our patient had flexion contracture of the knees and elbows. Another common manifestation of ARC syndrome is cholestatic jaundice and hepatomegaly. In previous reports, liver disease was accompanied with normal GGT, mild elevation of the liver enzymes (AST or ALT) and direct hyperbilirubinemia. Most of them showed evidence of biliary obstruction or severe intrahepatic cholestasis. One infant developed acute liver failure at the age of 6 weeks during an episode of sepsis. Findings of liver biopsy may include cholestasis with multinucleate giant cell change of hepatocytes, intrahepatic biliary hypoplasia and lipofuscin deposition [9]. Liver biopsy of our patient showed lobular disarray, giant cell transformation of hepatocytes, severe cholestasis and granular type brown-yellowish pigments in hepato- 
cytes. Our patient developed liver failure at age of 4 months, presenting with coagulopathy, hypoalbuminemia and elevated liver enzymes which were the cause of death.

The third manifestation of ARC syndrome is renal problem. Renal involvement in ARC patients is complex. Many abnormalities include glomerular involvement, proteinuria, hypernatremic dehydration, nephrogenic diabetes insipidus (NDI), dysplastic kidney and renal tubular acidosis (RTA) as described for these patients [2]. Nephrocalcinosis in our patient was one of our purposes in presenting this case. Our case presented with normal anion gap metabolic acidosis, hypokalemia, proteinuria and hypercalciuria that are manifestations of RTA. In addition, our patient had medullary nephrocalcinosis and secondary nephrogenic diabetes insipidus. It was manifested with hypernatremic dehydration, polyuria and low urine specific gravity. It was reported only in two cases of ARC syndrome [2].

One unique presentation of our case was congenital anomaly in his toes. He had four toes in each foot and abnormal overriding of the first and forth toes on the second and third ones. The only dysmorphic feature of the limbs that was previously reported in a Pakistani ARC patient was large hands and proximally inserted thumbs [4], but no lower limb anomaly has been reported for ARC syndrome till now. Another interesting finding in our patient was congenital hypothyroidism, which was treated with levothyroxin. Hypothyroidism was reported in three cases of ARC syndrome [4,5].
All of them were primarily of congenital type and treated with low doses of levothyroxin. Our patient also had low-set ears, which was previously reported in some cases $[4,5]$.

Ichthyosis is another presentation of our patient, as reported in 17 cases of ARC syndrome. Choi

. suggested that the defective secretion of the lamellar bodies in the epidermis, which might be mediated by sensory nerve action potential receptor protein complex, might be the cause of the ichthyosis phenotype in ARC syndrome [10]. Our patient did not have brain or hematologic manifestations.

\section{Conclusion}

In conclusion, ARC syndrome has various clinical manifestations. Lower limb congenital anomaly, hypothyroidism, liver failure and nephrocalcinosis are other rare presentations of ARC syndrome, which was manifested in our patient.

\section{Acknowledgement}

Authors would like to thank Dr. Nasrin Shokrpour for editorial assistance at Center for Development of Clinical Research of Namazi Hospital.

\section{Conflict of Interests}

There is no conflict of interests between authors.

\section{References}

1. Horslen SP, Quarrell OW, Tanner MS. Liver histology in the arthrogryposis multiplex congenita, renal dysfunction, and cholestasis (ARC) syndrome: report of three new cases and review. J Med Genet. 1994; 31(1):62-4.

2. Malaki M, Mandana R, Ghaffari S. ARC syndrome with complex renal problems: nephrocalcinosis, proximal and hyperkalemic distal RTA and nephrogenic diabetes insipidus. Saudi J Kidney Dis Transpl. 2012; 23(4):804-9

3. Saadah OI, Bokhari BE, Alshaeri TM, Jastaniah W. Haematological manifestations of arthrogryposisrenal dysfunction-cholestasis (ARC) syndrome: a case report. Arab J Gastroenterol. 2013; 14(1):26-8. 
4. Gissen P, Tee L, Johnson CA, Genin E, Caliebe A, Chitayat D, et al. Clinical and molecular genetic features of ARC syndrome. Hum Genet. 2006; 120(3):396-409.

5. Arhan E, Yusufoğlu AM, Sayli TR. Arc syndrome without arthrogryposis, with hip dislocation and renal glomerulocystic appearance: a case report. Eur J Pediatr. 2009; 168(8):995-8.

6. Tekin N, Durmuş-Aydoğdu S, Dinleyici EC, Bör O, Bildirici K, Akşit A. Clinical and pathological aspects of ARC (arthrogryposis, renal dysfunction and cholestasis) syndrome in two siblings. Turk J Pediatr. 2005; 47(1):67-70

7. Smith H, Galmes R, Gogolina E, Straatman-Iwanowska A, Reay K, Banushi B, et al.. Associations among genotype, clinical phenotype, and intracellular localization of trafficking proteins in ARC syndrome. Hum Mutat. 2012; 33(12):1656-64

8. Bull LN, Mahmoodi V, Baker AJ, Jones R, Strautnieks SS, Thompson RJ, Knisely AS. VPS33B mutation with ichthyosis, cholestasis, and renal dysfunction but without arthrogryposis: incomplete ARC syndrome phenotype. J Pediatr. 2006; 148(2):269-71.

9. Eastham K, McKiernan P, Milford D, Ramani P, Wyllie J, van't H, et al. ARC syndrome: an expanding range of phenotypes. Arch Dis Child. 2001; 85(5):415-20.

10. Choi HJ, Lee MW, Choi JH, Moon KC, Koh JK. Ichthyosis associated with ARC syndrome: ARC syndrome is one of the differential diagnoses of ichthyosis. Pediatr Dermatol. 2005; 22(6):539-42. 